\title{
The First Two Rounds of MD4 are Not One-Way
}

\author{
Extended Abstract
}

\author{
Hans Dobbertin \\ German Information Security Agency \\ P. O. Box 200363 \\ D-53133 Bonn \\ dobbertin@skom.rhein.de
}

In 1] it was shown that there are very effective attacks leading to collisions for the hash function MD4 designed by R. Rivest [3]. A summary of the status of hash functions of the MD4-family with respect to collision-resistence can be found in [2] and [4. However, attacking the one-wayness of a hash function is a much more demanding challenge, and in case of success it has much more devastating consequences. No result along this line is known for MD4 and its successors. Therefore it is worth to explore how the recently developed new analytic methods for finding collisions can be applied to construct preimages or second preimages. As a first step, we state here the following partial result:

Denote by MD4 ${ }^{[12]}$ the reduced version of MD4, where the third round of its underlying three-round compression function is cancelled, but everything else of its specification is kept (e.g. initial value, padding rule).

$$
\mathrm{MD}^{[12]} \text { is not one-way. }
$$

It takes less than one hour to find preimages on a PC. Second preimages take a few minutes or even less than a millisecond if the initial value is free.

Example. Assume we want to find a preimage of

$$
V=0 \times 00000000 \quad 0 \times 00000000 \quad 0 \times 00000000 \quad 0 \times 00000000 .
$$

We have constructed the following message $M=M_{0}\left\|M_{1}\right\| \ldots \| M_{28}$, which is hashed to this value $V$ by MD4 ${ }^{[12]}$ :

$$
\begin{aligned}
& M_{0}=0 \times 185 \mathrm{~B} 6 \mathrm{AC} 17 \quad M_{8}=0 \mathrm{xFF} 9405 \mathrm{C} 3 \quad M_{16}=0 \mathrm{x} 814 \mathrm{~F} 4825 \quad M_{24}=0 \times 847064 \mathrm{AD} \\
& M_{1}=0 \times 1856 \mathrm{AC} 17 \quad M_{9}=0 \mathrm{xFF} 9405 \mathrm{C} 3 \quad M_{17}=0 \mathrm{x} 814 \mathrm{~F} 4825 \quad M_{25}=0 \mathrm{x} 05 \mathrm{DDD} 0 \mathrm{~F} 5
\end{aligned}
$$

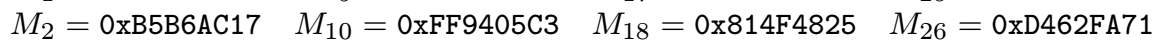

$$
\begin{aligned}
& M_{3}=0 \times 85574 \mathrm{~A} 58 \quad M_{11}=0 \times 126 \mathrm{EA} 1 \mathrm{D} 5 \quad M_{19}=0 \times 814 \mathrm{~F} 4825 \quad M_{27}=0 \times 56 \mathrm{~A} 79 \mathrm{DEC} \\
& M_{4}=0 \times 4353212 \mathrm{D} \quad M_{12}=0 \times 015 \mathrm{CB} 5 \mathrm{DO} \quad M_{20}=0 \times 9919 \mathrm{C} 508 \quad M_{28}=0 \times 00000080 \\
& M_{5}=0 \times 4353212 \mathrm{D} \quad M_{13}=0 \times 81 \mathrm{BBD} 193 \quad M_{21}=0 \times 9919 \mathrm{C} 508 \\
& M_{6}=0 \times 4353212 \mathrm{D} \quad M_{14}=0 \times 1 \mathrm{DEF} 9763 \quad M_{22}=0 \times 9919 \mathrm{C} 508 \\
& M_{7}=0 \times 3 \mathrm{E} 30333 \mathrm{E} \quad M_{15}=0 \times \mathrm{ADE} 9028 \mathrm{~B} \quad M_{23}=0 \times 2 \mathrm{FD} 7 \mathrm{BOF} 9
\end{aligned}
$$

We anticipate that a similar attack works for the last two rounds of MD4.

S. Vaudenay (Ed.): Fast Software Encryption - FSE'98, LNCS 1372, pp. 284-292 1998.

(C) Springer-Verlag Berlin Heidelberg 1998 
Technical Details for Checking the Example. According to the padding rule, before processing, a message has to be extended by a bit string

$$
P=100 \ldots 0(\text { bin }) \| \ell
$$

where $\ell$ is the 64-bit representation of the bit-length of the (unextended) message. In $P$, between 1 on the left and $\ell$ on the right side, the minimal number of zeros is placed such that the bit-length of the extended message becomes a multiple of $512=16 \times 32$ (to allow an iterative application of the compression function, which takes 16 words as input). The above $M$ has bit-length $29 \times 32$. This means that

$$
\ell=00000000000003 \mathrm{~A} 0(\text { hex })
$$

and $P$ is a 96 -bit string with the little-endian representation $P=P_{0}\left\|P_{1}\right\| P_{2}$ :

$$
\begin{aligned}
& P_{0}=0 \times 00000080 \\
& P_{1}=0 \times 000003 \mathrm{~A} 0 \\
& P_{2}=0 \times 00000000 .
\end{aligned}
$$

Denote by MD4 ${ }^{[12]}$-Compress the compression function of $\mathrm{MD}^{[12]}$, i.e. the first two rounds of the MD4 compression function. In order to compute the MD4 ${ }^{[12]}$ hash value of $M$, first MD4 ${ }^{[12]}$-Compress is applied to $M_{0}, \ldots, M_{15}$ with the following fixed initial value, which is a part of the specification of MD4:

$$
I V=0 \times 67452310 \quad \text { 0xEFCDAB89 0x98BADCFE } 0 \times 10325476 .
$$

This gives the output

$$
\begin{aligned}
C & =\mathrm{MD}^{[12]}{ }^{[1-C o m p r e s s}\left(I V ; M_{0}, \ldots, M_{15}\right) \\
& =\text { 0xA86FDECC } 0 x 25 B F 84 \text { C } 0 \text { 0xDB95C842 0xD0B260B9. }
\end{aligned}
$$

$C$ is then the initial value for the second application of $\mathrm{MD}^{[12]}{ }^{12}$ Compress with $M_{16}, \ldots, M_{28}, P_{0}, P_{1}, P_{2}$ as input. The output is the hash value of $M$ :

$$
\begin{aligned}
\operatorname{MD}^{[12]}(M) & =\mathrm{MD}^{[12]}-\operatorname{Compress}\left(C ; M_{16}, \ldots, M_{28}, P_{0}, P_{1}, P_{2}\right) \\
& =0 \mathrm{x} 000000000 \mathrm{x} 00000000 \text { 0x00000000 } 0 x 00000000 .
\end{aligned}
$$

\section{How to invert the first two rounds of MD4 compression}

Suppose $I V=\left(I V_{0}, I V_{1}, I V_{2}, I V_{3}\right)$ and the compress value $C=\left(C_{0}, C_{1}, C_{2}, C_{3}\right)$ are given. Set $H=\left(H_{0}, H_{1}, H_{2}, H_{3}\right):=C-I V$. Our approach is described in the following tables (the underlined intries are those which are up-dated in the repsective steps): 


\begin{tabular}{|c||c|c|c|c||c|}
\hline input & register A & register B & register C & register D & step \\
\hline \hline & $I V_{0}$ & $I V_{1}$ & $I V_{2}$ & $I V_{3}$ & \\
\hline$X_{0}$ & $*$ & $I V_{1}$ & $I V_{2}$ & $I V_{3}$ & step 0 \\
\hline$X_{1}$ & $*$ & $I V_{1}$ & $I V_{2}$ & $\underline{*}$ & step 1 \\
\hline$X_{2}$ & $*$ & $I V_{1}$ & $\underline{*}$ & $*$ & step 2 \\
\hline$X_{3}$ & $*$ & $\underline{*}$ & $*$ & $*$ & step 3 \\
\hline$X_{4}$ & $\underline{*}$ & $*$ & $*$ & $*$ & step 4 \\
\hline$X_{5}$ & $*$ & $*$ & $*$ & $\underline{*}$ & step 5 \\
\hline$X_{6}$ & $*$ & $*$ & $\underline{*}$ & $*$ & step 6 \\
\hline$X_{7}$ & $*$ & $\underline{*}$ & $*$ & $*$ & step 7 \\
\hline$X_{8}$ & $\underline{P_{0}}$ & $*$ & $*$ & $*$ & step 8 \\
\hline$X_{9}$ & $P_{0}$ & $*$ & $*$ & $\underline{P_{3}}$ & step 9 \\
\hline$X_{10}$ & $P_{0}$ & $*$ & $\underline{P_{2}}$ & $P_{3}$ & step 10 \\
\hline$X_{11}$ & $P_{0}$ & $P_{1}$ & $P_{2}$ & $P_{3}$ & step 11 \\
\hline$X_{12}$ & $\underline{K}$ & $P_{1}$ & $P_{2}$ & $P_{3}$ & step 12 \\
\hline$X_{13}$ & $K$ & $P_{1}$ & $P_{2}$ & $\underline{K}$ & step 13 \\
\hline$X_{14}$ & $K$ & $P_{1}$ & $\underline{K}$ & $K$ & step 14 \\
\hline$X_{15}$ & $K$ & $B_{3}$ & $K$ & $K$ & step 15 \\
\hline
\end{tabular}

Round Two OF MD4 COMPRESSION

\begin{tabular}{|c||c|c|c|c||c|}
\hline input & register A & register B & register C & register D & step \\
\hline \hline$X_{0}$ & $\underline{K}$ & $B_{3}$ & $K$ & $K$ & step 16 \\
\hline$X_{4}$ & $K$ & $B_{3}$ & $K$ & $\underline{K}$ & step 17 \\
\hline$X_{8}$ & $K$ & $B_{3}$ & $\underline{K}$ & $K$ & step 18 \\
\hline$X_{12}$ & $K$ & $\underline{B_{2}}$ & $K$ & $K$ & step 19 \\
\hline$X_{1}$ & $\underline{K}$ & $B_{2}$ & $K$ & $K$ & step 20 \\
\hline$X_{5}$ & $K$ & $B_{2}$ & $K$ & $\underline{K}$ & step 21 \\
\hline$X_{9}$ & $K$ & $B_{2}$ & $\underline{K}$ & $K$ & step 22 \\
\hline$X_{13}$ & $K$ & $B_{1}$ & $K$ & $K$ & step 23 \\
\hline$X_{2}$ & $\underline{K}$ & $B_{1}$ & $K$ & $K$ & step 24 \\
\hline$X_{6}$ & $K$ & $B_{1}$ & $K$ & $\underline{K}$ & step 25 \\
\hline$X_{10}$ & $K$ & $B_{1}$ & $\underline{K}$ & $K$ & step 26 \\
\hline$X_{14}$ & $K$ & $\underline{B_{0}}$ & $K$ & $K$ & step 27 \\
\hline$X_{3}$ & $H_{0}$ & $B_{0}$ & $K$ & $K$ & step 28 \\
\hline$X_{7}$ & $H_{0}$ & $B_{0}$ & $K$ & $\underline{K}$ & step 29 \\
\hline$X_{11}$ & $H_{0}$ & $B_{0}$ & $H_{2}$ & $H_{3}$ & step 30 \\
\hline$X_{15}$ & $H_{0}$ & $H_{1}$ & $H_{2}$ & $H_{3}$ & step 31 \\
\hline
\end{tabular}

That is, we assume that the contents of the A-, D-, and C-registers equals a constant $K$ in steps $12,16,20,24$, steps $13,17,21,25$, and steps $14,18,22,26$, respectively.

Idea. In round two the majority function $\mathrm{g}$ is applied, and by the described approach we can separate the contents in the B-registers from those in the other registers in round two. 
Algorithm. Choose $K$ and $B_{0}$ randomly. Now $X_{0}, \ldots, X_{11}$, and $X_{15}$ are fixed by the steps $16,20,24,28,17,21,25,29,18,22,26,30$, and 31 , respectively: for instance in step 16 we have the equation

$$
K=\left(K+g(B 3, K, K)+X_{0}+K_{1}\right)<<3=\left(K+K+X_{0}+K_{1}\right)<<3
$$

with $K_{1}=0 x 5$ A827999. Thus $X_{0}=(K<<29)-2 K-K_{1}$, and so on.

Now compute $P_{0}, P_{1}, P_{2}, P_{3}$ by applying $X_{0}, \ldots, X_{11}$ in step $0, \ldots, 11$. The values $P_{0}, P_{1}, P_{2}, P_{3}$ allow next to determine $X_{12}, X_{13}, X_{14}$ from the steps $12,13,14$.

Use $X_{15}$ in step 15 to compute $B_{3}, X_{12}$ in step 19 to compute $B_{2}, X_{13}$ in step 23 to compute $B_{1}$, and finally $X_{14}$ in step 27 to compute another value for $B_{0}$ which is "derived from above". If the latter $B_{0}$-value matches the chosen one, then we have found a preimage, otherwise try again.

Thus we need about $2^{32}$ trials to be successful. However, the algorithm can be speeded up by a factor of 100 or more if we use "continuous approximation" for the computation of $B_{0}$ (where $K$ is fixed respectively, in order to have sufficient continuity; see second part of the below C-progam).

\section{References}

1. H. Dobbertin, Cryptanalysis of MD4, Fast Software Encryption (Third Workshop on Cryptographic Algorithms, Cambridge 1996), Lecture Notes in Computer Science, Springer-Verlag 1996, pp. 55-72.

2. H. Dobbertin, The status of MD5 after a recent attack, CryptoBytes, The technical newsletter of RSA Laboratories, vol. 2/2, Sommer 1996, pp. 1-6.

3. R. Rivest, The MD4 message-digest algorithm, Request for Comments (RFC) 1320, Internet Activities Board, Internet Privacy Task Force, April 1992.

4. M.J.B. Robshaw, On recent results for MD2, MD4 and MD5, Bulletin 4, RSA Laboratories, November 1996 (see http://www.rsa.com/PUBS/).

\section{Appendix}

\section{C-program inverting the reduced MD4 with cancelled third compression round}

The first part of the program below is a modifiction of the above algorithm, which allows to match the redundancy in the input required by the padding rule. On a Pentium PC the program finds a hash-preimage in about 15-20 minutes on the average.

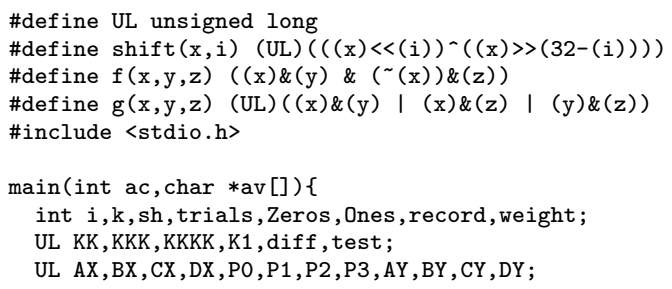


UL $\mathrm{X} 0, \mathrm{X} 1, \mathrm{X} 2, \mathrm{X} 3, \mathrm{X} 4, \mathrm{X} 5, \mathrm{X} 6, \mathrm{X} 7, \mathrm{X} 8, \mathrm{X} 9, \mathrm{X} 10, \mathrm{X} 11, \mathrm{X} 12, \mathrm{X} 13, \mathrm{X} 14, \mathrm{X} 15$;

UL Delta_A,Delta_BO,BO_basic,P2_basic;

UL AA, B0, B1, B2 , B3, C0, C1, C2, C3;

UL HO, H1 , H2 , H3, HHO, HH1 , HH2 , HH3, HHHO , HHH1 , HHH2 , HHH3;

UL Q0,Q1,Q2, Q3, IV0,IV1, IV2, IV3;

UL MO,M1,M2,M3,M4, M5 , M6, M7 ,M8, M9, M10;

UL M11,M12,M13,M14,M15,M16,M17,M18,M19;

UL M20,M21, M22, M23, M24, M25, M26, M27, M28;

UL PP0,PP1,PP2;

if $(\mathrm{ac} !=2)\{$

fprintf (stdout, "Usage: \%s seed \n", av [0]); exit (1);

srand (atoi (av [1]));

$\mathrm{K} 1=0 \times 5 \mathrm{~A} 827999 ;$

$\mathrm{KK}=0 \times 57902134$

$\mathrm{KKK}=0 \times 57902134$;

/* We have here a special case of a more general algorithm. In general KK and KKK are different, but have only a small Hamming difference. How to choose these constants will be explained in the complete paper about this attack.*/

Zeros $=0$;

Ones $=0$;

trials $=0$;

/* Here you can specify the hash value (HHO, HH1, HH2, HH3) */ $\mathrm{HHO}=0 \times 0$;

$\mathrm{HH} 1=0 \mathrm{x} 0$

$\mathrm{HH} 2=0 \times 0$

$\mathrm{HH} 3=0 \times 0$;

$/ * * * * * * * * * * * * * * * * * * * * * * * * * * * * * * * * * * * * * * * * * * * * * * * * * * * * * * * * * * * * * *) /$

НHHO=HHO;

$\mathrm{HHH} 1=\mathrm{HH} 1$;

$\mathrm{HHH} 2=\mathrm{HH} 2$;

HHH $3=$ HH 3 ;

/* Here starts the first part: searching M16, ., M28 */

START_I :

record $=33$;

$\mathrm{AA}=\mathrm{KK}$;

$\mathrm{H} 2=\operatorname{rand}()$;

H3 $=\operatorname{rand}() \& 0 x f f f f f f 7 f ;$

$\mathrm{HO}=\mathrm{KK}$;

$\mathrm{X} 15=0 \mathrm{x} 0$;

$\mathrm{P} 1=\operatorname{shift}(\mathrm{KK}, 13)-\mathrm{KK}-\mathrm{X} 15$;

$\mathrm{X} 14=0 \times 3 \mathrm{~A} 0$;

$\mathrm{KKKK}=\operatorname{shift}(\mathrm{KKK}+\mathrm{KK}+\mathrm{X} 14+\mathrm{K} 1,13)$;

$\mathrm{H} 1=\operatorname{shift}(\mathrm{KKKK}+\mathrm{g}(\mathrm{H} 2, \mathrm{H} 3, \mathrm{KK})+\mathrm{X} 15+\mathrm{K} 1,13)$;

IVO=HHO-HO;

IV $1=\mathrm{HH} 1-\mathrm{H} 1$;

IV $2=\mathrm{HH} 2-\mathrm{H} 2$;

IV $3=$ HH $3-\mathrm{H} 3$;

$\mathrm{XO}=\operatorname{shift}(\mathrm{KK}, 29)-\mathrm{AA}-\mathrm{KK}-\mathrm{K} 1 ;$

$\mathrm{X} 1=\operatorname{shift}(\mathrm{KK}, 29)-\mathrm{KK}-\mathrm{KK}-\mathrm{K} 1$;

$\mathrm{X} 2=\mathrm{X} 1$;

$\mathrm{x} 3=\mathrm{x} 1$;

$\mathrm{X} 4=\operatorname{shift}(\mathrm{KK}, 27)-\mathrm{KK}-\mathrm{KK}-\mathrm{K} 1$; 


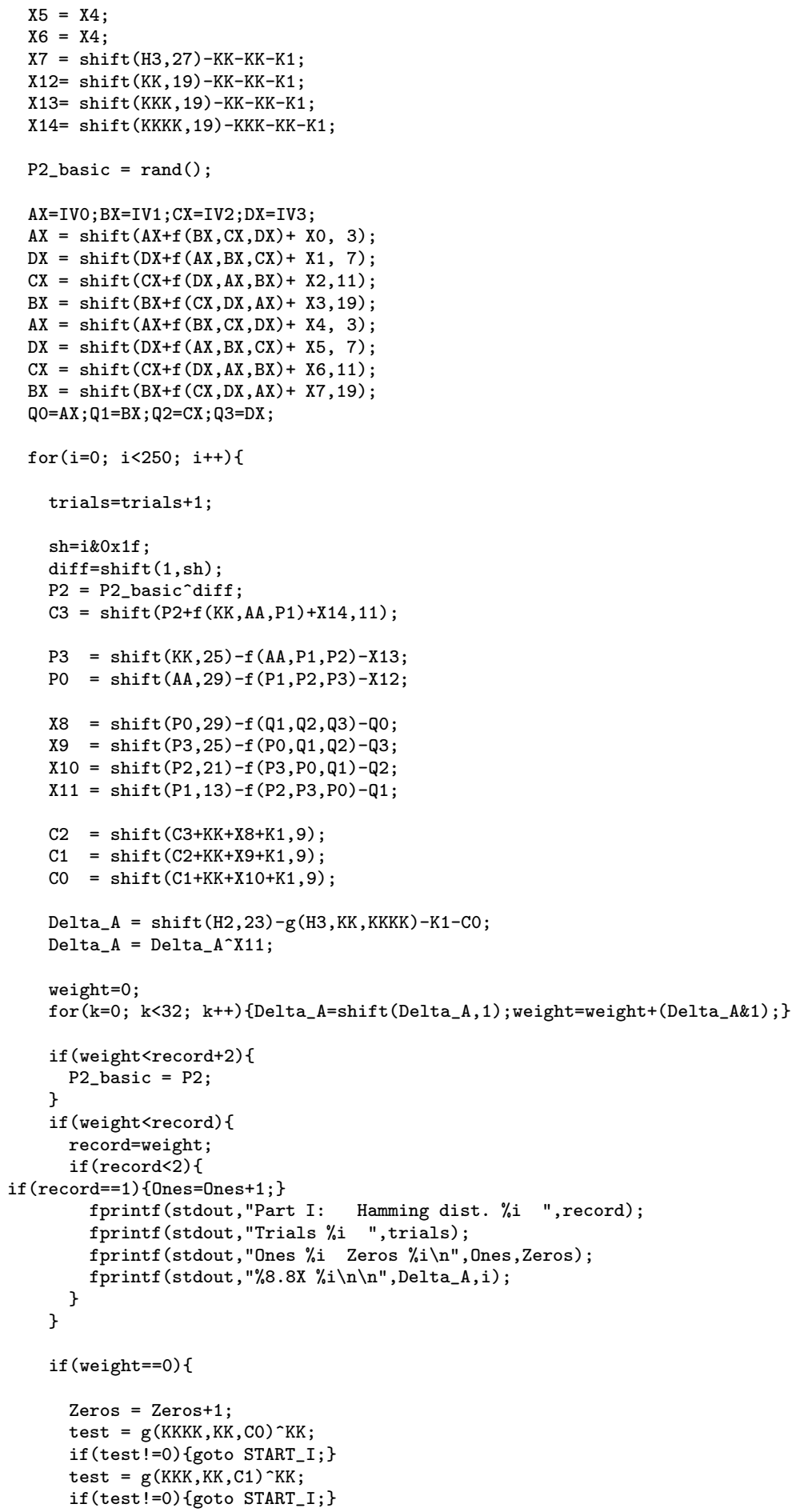




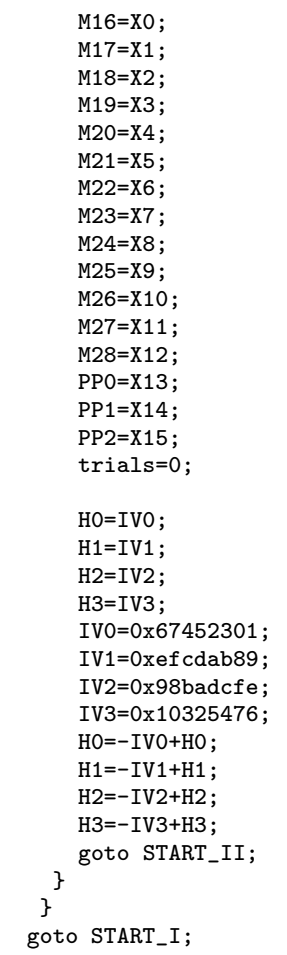

/* Here starts the second part: searching M0,.,, M15 */

START_II:

record $=33$;

$\mathrm{KK}=\operatorname{rand}()$;

B0_basic=rand ();

$\mathrm{XO}=\operatorname{shift}(\mathrm{KK}, 29)-\mathrm{KK}-\mathrm{KK}-\mathrm{K} 1$;

$\mathrm{X} 1=\operatorname{shift}(\mathrm{KK}, 29)-\mathrm{KK}-\mathrm{KK}-\mathrm{K} 1$;

$\mathrm{X} 2=\operatorname{shift}(\mathrm{KK}, 29)-\mathrm{KK}-\mathrm{KK}-\mathrm{K} 1$;

$\mathrm{X} 3=\operatorname{shift}(\mathrm{HO}, 29)-\mathrm{KK}-\mathrm{KK}-\mathrm{K} 1$;

$\mathrm{X} 4=\operatorname{shift}(\mathrm{KK}, 27)-\mathrm{KK}-\mathrm{KK}-\mathrm{K} 1$;

$\mathrm{X} 5=\operatorname{shift}(\mathrm{KK}, 27)-\mathrm{KK}-\mathrm{KK}-\mathrm{K} 1$;

$\mathrm{X} 6=\operatorname{shift}(\mathrm{KK}, 27)-\mathrm{KK}-\mathrm{KK}-\mathrm{K} 1$;

$\mathrm{X} 8=\operatorname{shift}(\mathrm{KK}, 23)-\mathrm{KK}-\mathrm{KK}-\mathrm{K} 1$;

$\mathrm{X} 9=\operatorname{shift}(\mathrm{KK}, 23)-\mathrm{KK}-\mathrm{KK}-\mathrm{K} 1$;

$\mathrm{X} 10=\operatorname{shift}(\mathrm{KK}, 23)-\mathrm{KK}-\mathrm{KK}-\mathrm{K} 1$;

for $(i=0 ; i<250 ; i++)\{$

trials=trials +1

$\mathrm{sh}=\mathrm{i} \& 0 \mathrm{x} 1 \mathrm{f}$;

$\operatorname{diff}=\operatorname{shift}(1, \mathrm{sh})$;

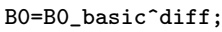

$\mathrm{X} 7=\operatorname{shift}(\mathrm{H} 3,27)-\mathrm{g}(\mathrm{HO}, \mathrm{BO}, \mathrm{KK})-\mathrm{KK}-\mathrm{K} 1$;

$\mathrm{X} 11=\operatorname{shift}(\mathrm{H} 2,23)-\mathrm{g}(\mathrm{H} 3, \mathrm{HO}, \mathrm{BO})-\mathrm{KK}-\mathrm{K} 1$;

$\mathrm{X} 15=\operatorname{shift}(\mathrm{H} 1,19)-\mathrm{g}(\mathrm{H} 2, \mathrm{H} 3, \mathrm{HO})-\mathrm{K} 1-\mathrm{BO}$; 


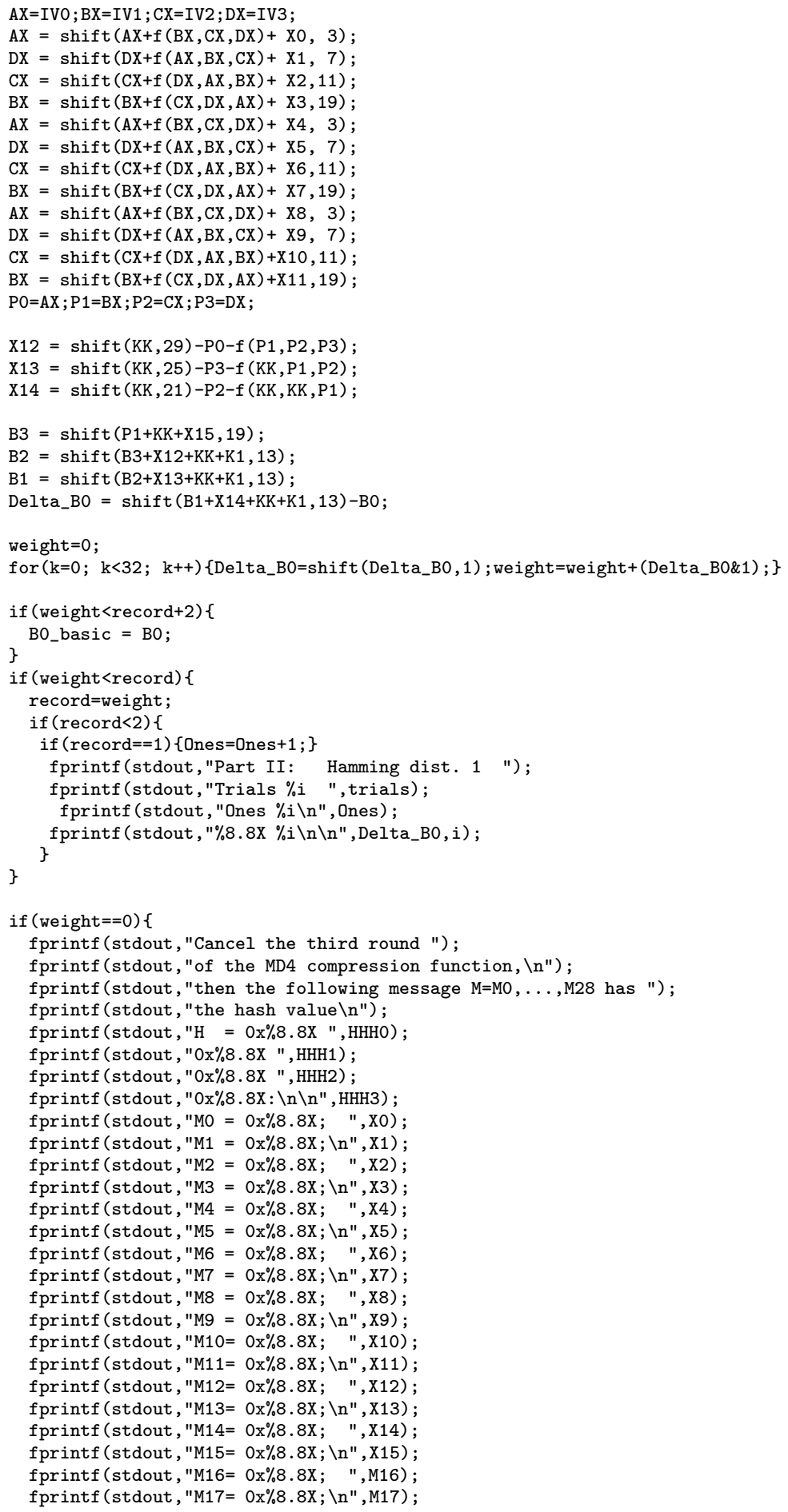




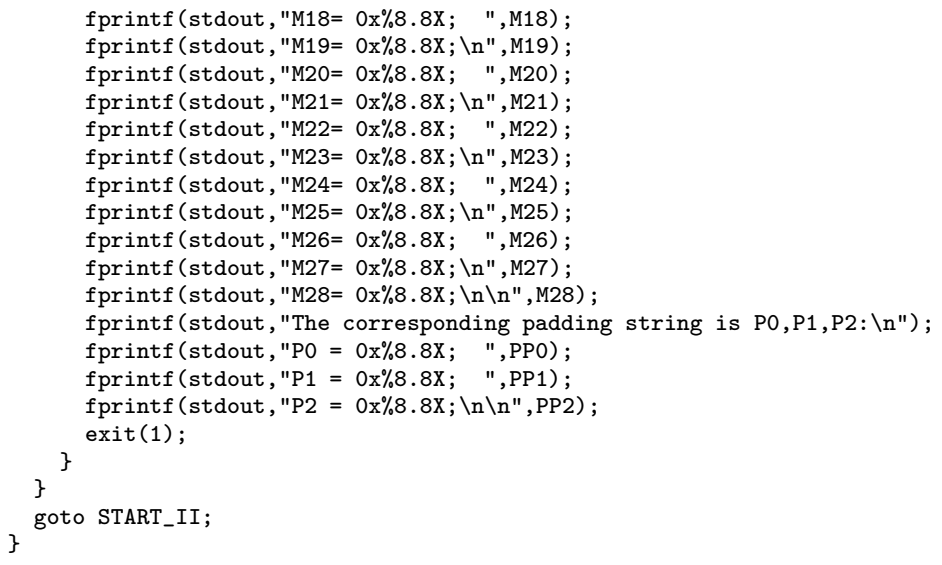

